\title{
1 Genome-wide CRISPR Screens Identify Ferroptosis as a 2 Novel Therapeutic Vulnerability in Acute Lymphoblastic 3 Leukemia
}

4 Marie-Eve Lalonde ${ }^{1}$, Marc Sasseville ${ }^{1}$, Anne-Marie Gélinas ${ }^{1}$, Jean-Sébastien Milanese ${ }^{1}$, Kathie 5 Béland $^{2}$, Simon Drouin ${ }^{1}$, Elie Haddad ${ }^{2}$ and Richard Marcotte ${ }^{1}$

${ }^{1}$ Human Health Therapeutics Research Centre, National Research Council Canada, Montréal, QC, H4P 2R2, Canada.

10 Correspondence: Richard Marcotte, National Research Council Canada, Building Montreal11 Royalmount, 6100 Royalmount avenue, Montréal, Qc, Canada, H4P 2R2. E-mail address:

$12 \quad$ richard.marcotte@nrc-cnrc.gc.ca

${ }^{2}$ Centre Hospitalier Universitaire Sainte-Justine, Université de Montréal, Montréal, QC, H3T 1C5, Canada.

Acute lymphoblastic leukemia (ALL) is the most frequent cancer diagnosed in children. Despite the great progress achieved over the last 40 years, with cure rates now exceeding $85 \%$, refractory or relapsed ALL still exhibit a dismal prognosis. This poor outcome reflects the lack of treatment options specifically targeting relapsed or refractory ALL. To address this gap, we have performed whole-genome CRISPR/Cas drop-out screens on a panel of seven B-ALL cell lines. Our results demonstrate that while there was a significant overlap in gene essentiality between ALL cell lines and other cancer types survival of ALL cell lines was dependent on several unique metabolic pathways, including an exquisite sensitivity to GPX4 depletion and ferroptosis induction and GPX4 KO. Detailed molecular analysis of B-ALL cells suggest that they are primed to undergo

24 ferroptosis as they exhibit high steady-state oxidative stress potential, a low buffering capacity,

25 and a disabled GPX-independent secondary lipid peroxidation detoxification pathway. Finally, we

26 validated the sensitivity of B-ALL to ferroptosis induction using patient-derived B-ALL samples.

\section{Introduction}

28 Acute lymphoblastic leukemia (ALL) is the most prevalent cancer during childhood, representing 29 nearly $80 \%$ of all cancer in this age group ${ }^{1}$. Treatment protocols have greatly improved over the 
30 last 30 years, such that the survival rate reaches $>85 \%{ }^{2}$. Despite this therapeutic success, the 31 prognosis for relapsed patients remains dismal with a less than 50\% 5 year survival, still making 32 ALL the second highest cause of death by disease amongst children in Canada and in the U.S 33 (Vrooman and Silverman 2016). In addition, up to 65-70\% of pediatric ALL survivors will suffer 34 from long-term debilitating or even life-threatening treatment related sequelae ${ }^{3,4}$. Hence, novel 35 therapeutic avenues that are both more effective at achieving long-term remission while eliciting 36 less acute long-term toxicities than current treatment regimen are required to treat these patients.

37 Recent advances in the use of clustered regularly-interspaced short palindromic repeat 38 (CRISPR)/Cas9 technology has revolutionized functional genomics and the analysis of gene 39 function in mammalian cells with its precision, ease of use, speed, and versatility. Whole-genome 40 bulk pooled CRISPR screens, using multiple sgRNAs targeting each human gene in a single 41 CRISPR library, have allowed the identification of genes implicated in processes underlying 42 phenotypic read-outs such as proliferation and survival in hundreds of tumoral cell lines ${ }^{5-7}$. These 43 large datasets revealed panels of core essential and pan-cancer genes and unraveled key players 44 required for cell viability/proliferation in cell lines derived from multiple histotypes. Although 45 ALL is a common form of cancer, very few ALL cell lines have been previously screened by large 46 dropout screen studies ${ }^{5-7}$. This is likely explained by the difficulty in infecting pre-B and $\mathrm{T}$ 47 lymphocytes with lentiviruses, a technical requirement for performing whole-genome CRISPR screens.

In this report, we performed whole-genome dropout CRISPR screens of seven B-ALL cell lines.

50 These screens revealed a surprisingly large subset of essential genes unique to ALL cell survival 51 that were not reported to be core essential genes in previous studies ${ }^{6-8}$. These B-ALL-enriched 52 genes were implicated in different cellular pathways and functions, many of which were associated 53 directly or indirectly to ferroptosis. In recent years, this non apoptotic pathological cell death has 54 gained increasing attention in cancer research, particularly for its potential tumor-suppressor 55 function that could be exploited for neoplastic disease treatment ${ }^{9-11}$. By using different cellular 56 and molecular biology approaches, we validate that B-ALL cell lines have an exquisite 57 vulnerability to ferroptosis induction. This sensitivity is illustrated by the extreme responsiveness 58 of cells to glutathione peroxidase 4 (GPX4) inhibition, but also to other pathways that regulate 59 GPX4 activity. We also show that this sensitivity is rescued by exogenous expression of FSP1, a 
60 recently characterized ferroptosis inhibitor ${ }^{12,13}$ that we found to be poorly expressed, not only in

61 B-ALL cells, but in leukemias in general. Finally, we demonstrate that this acute sensitivity to

62 ferroptosis induction is also observed in a panel of B-ALL patient-derived tumor samples.

\section{Results}

64 Whole-genome CRISPR screens of B-ALL cell lines

65 In order to identify genes that are essential for B-ALL cells, we performed whole-genome pooled 66 dropout CRISPR screens using a two-vector system (Fig. 1A) ${ }^{14}$. First, a stable pool of Cas9-

67 expressing cells was established for each cell line. This preliminary step was limiting for many 68 ALL cell lines, since several pools did not reach the 75\% Cas9 activity threshold required for 69 further screening (Suppl. Table 2). For some of these cell lines, Cas9 activity decreased rapidly 70 after blasticidin selection, particularly in T-ALL cell lines (data not shown), suggesting that 71 constitutive Cas9 expression might be toxic, similar to what is reported in AML cell lines ${ }^{14}$. Of all 72 the pools tested, the 7 B-ALL Cas9-stable pools attaining $>75 \%$ Cas9 activity were infected with 73 a whole-genome lentiviral 90K single-guided RNA (sgRNA) library (Suppl. Fig. 1A and B) ${ }^{14}$. 74 Screen quality was very high, as shown by the BAGEL essential and non-essential genes ${ }^{8,15}$ 75 comparison precision/recall curves (Suppl. Fig. 1C). Furthermore, principal component analysis 76 (PCA) shows that T0 time points from all cell lines are tightly clustered and, despite some variance 77 shown in the final timepoint (Tf) between cell lines, cell line replicates were tightly clustered and 78 significantly different from T0, suggesting that significant dropout was achieved in the Tf samples 79 (Suppl. Fig. 1D).

\section{Whole-genome CRISPR screens identify vulnerabilities specific to B-ALL}

81 In an attempt to identify essential gene unique to B-ALL, we compared the essential genes 82 identified in the screened B-ALL cell lines with the list of essential genes reported in previous 83 datasets. We noticed a considerable overlap of B-ALL essential genes with Broad and Sanger core 84 essential genes $^{7}$ (Fig. 1B and C, Suppl. Table 3 and 4). 1204 essential genes were identified in at 85 least 4/7 B-ALL cell lines (FDR $<0.05$ ), of which $67 \%$ and $63 \%$ were also included in the Broad 86 and Sanger gene lists, respectively and, as expected, belong to core biological processes such as 87 the cell cycle, mRNA translation, splicing, and Pol II transcription- pathways that are vital for 
growth and survival of most cell lines. Strikingly, 72 genes were identified as essential in every B-ALL cell line screened but were not previously identified as essential genes in the Broad and Sanger datasets (Fig. 1B, green line). Likewise, 98 genes were previously defined as essential in both the Sanger and Broad dataset but were not essential in any of the ALL cell lines (Fig. 1B, red line). We defined as "B-ALL-enriched essential genes" all hits that were found in at least 4/7 of B-ALL cell lines, but that were not present in the Broad or Sanger essential gene datasets (a total of 319 genes as shown in Fig. 1C). These genes are enriched for ALL-associated transcription factors, such as $P A X 5, E B F 1, C B F B, T C F 3$, and $R U N X 1^{16-18}$, B-cell receptor and signaling, such as $C D 79 A, C D 79 B$ and PIK3CD (Chu and Arber 2001; Kruth et al. 2017; Serafin et al. 2019), and other known B-cell vulnerabilities, such as $C D K 6, C C N D 3$, and $B C L 2^{22-24}$ (Fig. 1D). A few genes were also enriched in screened ALL cell lines, such as TP53 (most B-ALL cell lines have wildtype TP53) and TBC1D4, indicating that $\mathrm{KO}$ of these genes provided a proliferative advantage (Fig. 1D, F, Suppl. Table 3). Mapping the "B-ALL-enriched essential genes" onto the STRING database revealed that these genes were members of specific functional subnetworks with multiple protein-protein interactions (Fig. 1E), such as RUNX1-transcriptional regulation, iron-sulfur cluster assembly, and regulation of lipid metabolism genes. WikiPathway enrichment analysis also identified glutathione metabolism, one carbon metabolism and pentose phosphate pathways, all of

105 which would impact ferroptosis, and ferroptosis regulation itself as specific pathways for B-ALL cell survival (Suppl. Table 5). Ferroptosis is an iron-dependent form of necrosis which is triggered

107 via lipid peroxidation of polyunsaturated fatty acid (PUFA) at the cell membrane ${ }^{25}$ and has 108 garnered significant interest in the past few years as an alternative drug-induced cell death 109 mechanisms to therapy-induced apoptosis-resistant cancers ${ }^{9}$. Central to this cell death pathway is 110 GPX4, one of the top-ranked essential gene according to our screen results (Fig. 1F, suppl. Table 1113 ) and is the main inhibitor of ferroptosis induction. Interestingly, multiple genes potentially 112 impacting GPX4 activity were included in "B-ALL-enriched essential genes" list (Fig. 1F). Hence, 113 our whole-genome screens of B-ALL cell lines identified several genes unique to this histotype 114 and enriched for specific functional pathways, several of which could directly impact ferroptosis 115 induction. 
117 GPX4, a selenocysteine-containing glutathione peroxidase, reduces phospholipid hydroperoxides

118 to lipid alcohol with the help of glutathione (GSH) as an obligate cofactor and acts as the main

119 endogenous inhibitor of ferroptosis induction ${ }^{26}$. To validate that inhibition of GPX4 induces

120 ferroptosis in ALL cells, we treated cells with RSL3, a direct inhibitor of GPX4 ${ }^{26}$. Comparison of

121 dose-response curves in B-ALL to non-ALL and GPX4 non-essential cell lines (A549, MCF7,

122 NCIH226) indicates that B-ALL cell lines are particularly sensitive to RSL3 treatment (Fig. 2A).

123 This sensitivity was significantly higher compared to the RSL3 sensitivity of many other cell lines

124 reported in previous studies ${ }^{12,27}$. To confirm that RSL3 treatment induce ferroptosis but not 125 apoptosis, we stained ALL cells with C11 BODIPYTM, which stains peroxidated lipids, and 126 Annexin V, respectively (Suppl. Fig. 2A). Lipid peroxidation, but not Annexin V staining, was 127 only detected in the RSL3-treated cells (Suppl. Fig. 2A). Notably, higher lipid peroxidation levels 128 were also found in steady-state conditions in B-ALL cells compared to non-ALL cells (Suppl. Fig. 129 2B). To confirm the sensitivity of ALL to ferroptosis induction genetically, we generated GPX4 130 knock-out (KO) clones using CRISPR/Cas9 by constantly growing targeted cells in the presence 131 of ferrostatin-1, a radical trapping agent, which strongly inhibits ferroptosis (Fig. 2B and C) ${ }^{25,27}$.

132 Withdrawal of the drug in GPX4 KO clones led to cell death within 18 hours after removal, which 133 confirms the rapid induction of cell death after GPX4 inhibition in B-ALL cells (Fig. 2B). RSL3 134 drug treatment could partially be rescued by ferrostatin-1, the iron chelator deferoxamine (DFO), 135 as well as PD146176, a 15-lipoxygenase inhibitor, and EUK-134, a general antioxidant (Fig. 2D), 136 but not with Z-VAD-FMK, a pan-inhibitor of caspases and apoptosis. These results indicate that 137 RSL3 creates a lethal oxidative stress environment that is, to some extent, iron-dependent. 138 Interestingly, only lower DFO concentration that was previously reported could rescue cell 139 viability ${ }^{25,27,28}$. This discrepancy can be explained by the fact that B-ALL cells are more sensitive 140 to elevated DFO treatment (Suppl. Fig. 2D), which could reflect their higher requirement for iron 141 for sustained proliferation compared to other normal cell types and malignancies ${ }^{29}$.

142 B-ALL cells are sensitive to perturbations in pathways regulating GPX4 activity

143 Because of its requirement for specific cellular metabolites, GPX4 activity and ferroptosis 144 induction are influenced by many metabolic pathways ${ }^{9}$. Several genes regulating these pathways 145 were identified within B-ALL-enriched essential genes (Fig.s 1F and 6). These include genes 146 implicated in the synthesis of GSH (SLC7A11/SLC3A2/GCLC), mevalonate 
(HMGCR/MVD/MVK), lipids (ASCL3/4, FASN, MCAT), selenocompounds (SEPSEC, EEFSEC, SEPHS2), and iron metabolism (PCPB2, TFRC, STEAP3).

Interestingly, most B-ALL cells showed significantly lower levels of SLC7A11 and GSH compared to non-ALL cells, in steady-state conditions (Fig. 3A and B). Because of its essential role for GPX4 activity and in oxidative stress protection, low GSH levels could contribute to the increased sensitivity of B-ALL to ferroptosis induction. Accordingly, B-ALL cell lines, with the exception of NALM6 whose sensitivity was intermediate, were more sensitive to buthionine sulfoximine (BSO), a glutamate-cysteine ligase (GCLC) inhibitor ${ }^{30}$, and to erastin (a direct inhibitor of the $\mathrm{Xc}^{-}$transporter responsible for L-cystine import), than non-ALL cell lines where GPX4 is non-essential (Fig. 3C, D and E). Rescue of BSO or treatments in NALM6 and RS4;11 cells by addition of exogenous GSH indicates that GSH synthesis is essential for ferroptosis inhibition in B-ALL lines (Fig. 3E, suppl. Fig. 3A).

The mevalonate pathway regulates lipid peroxidation by two different mechanisms. First, it produces CoQ10, which contributes to detoxification of lipid reactive oxygen species (ROS) ${ }^{31}$. Second, it regulates the production of Isopentenyl pyrophosphate (IPP), a metabolite required for Sec-tRNA (a tRNA depositing selenocysteine on proteins) synthesis ${ }^{31}$. Since GPX4 contains a selenocysteine residue essential for ferroptosis inhibition ${ }^{32}$, regulation of selenocysteine incorporation into GPX4 can directly influence its activity. B-ALL cells were more sensitive to inhibition of the mevalonate pathway using FIN56, a dual inhibitor of squalene synthase (an enzyme involved in cholesterol biosynthesis) ${ }^{33}$ and GPX4 in contrast to non-ALL cells (Fig. 3F, Suppl. Fig. 3A). Further confirming our observations, treatments of B-ALL cells with other mevalonate pathway inhibitors (lovastatin, simvastatin) also induced ferroptosis (Fig. 3G).

In an attempt to determine which genes identified as essential in our primary screens dropped out of the pool specifically because of ferroptosis induction, we performed whole-genome CRISPR screens in two Cas9-expressing B-ALL cell lines in the presence or absence of ferrostatin-1 (Suppl. Fig. 3B, Suppl. Table 6). In this setting, we would expect genes depleted in the untreated group because of ferroptosis induction to no longer being depleted in the ferrostatin-treated group. As expected, GPX4 sgRNA were no longer depleted in the presence of ferrostatin-1 and showed the highest fold change between untreated and treated samples (Fig. 3H). Furthermore, three genes 
176

177

178

179

180

181

182

183

184

185

186

187

188

189

190

191

192

193

194

195

196

197

198

199

200

201

202

203

204

implicated in selenocysteine synthesis (PSTK, EEFESEC and SEPSECS) were also in the top hits further arguing that selenocysteine levels regulate ferroptosis in B-ALL cells (Fig. 3G, Suppl.

Table 6). Cells in which these genes were individually knocked out using CRISPR/Cas9 also showed increased lipid peroxidation (Suppl. Fig. 3C), strongly supporting ferroptosis induction. These results are consistent with DepMap CRISPR screen data. By segregating cell lines as GPX4 essential or GPX4 non-essential (Suppl. Fig. 3D, see materials and methods) and looking for genes that are co-essential with GPX4, we observed co-essentiality of SEPSECS, SEPHS2, EEFSEC, PSTK and SECISBP2 genes (Suppl. Fig. 3E), which are all selenocompound metabolism genes.

\section{B-ALL cells express low levels of FSP1, a potent ferroptosis inhibitor}

Distribution of GPX4 mRNA expression and protein levels between GPX4 sensitive and nonsensitive cell lines in the DepMap/CCLE indicate that GPX4 expression level does not account for GPX4 essentiality in cells (Suppl. Fig. 4A and B). When looking at differentially expressed genes between these two groups we noticed that FSP1/AIFM2, a recently characterized ferroptosis inhibitor $^{12,13}$, was expressed at lower levels in cells that were dependent on GPX4 compared to non-GPX4 essential cells (Suppl. Fig. 4C). A similar trend was also seen at the protein level (Fig. 4A). Moreover, mRNA and protein levels of FSP1 were particularly low in leukemia cell lines and could not be detected by Western Blot (Fig. 4A-B, Suppl. Fig. 4C). Thus, this low FSP1 level could potentially contribute to the acute vulnerability of B-ALL cells to ferroptosis induction. To test this hypothesis, we generated B-ALL stable pools that overexpressed FSP1 (Fig. 4B) and compare their RSL3 dose-response curve with parental cells (Fig. 4C). Both pools (low or high) overexpressing FSP1 rescued sensitivity to RSL3 treatment by approximately ten-fold compared with parental cell lines, suggesting that the low endogenous constitutive levels of FSP1 contribute to the ferroptosis sensitivity in B-ALL. Furthermore, rescue levels being independent of FSP1 overexpression level indicate that weak overexpression is sufficient to inhibit ferroptosis.

\section{Primary ALL PDX are also sensitive to ferroptosis induction}

To validate that the sensitivity observed in B-ALL cell lines is conserved in patient-derived samples, we tested different ferroptosis inducing drugs on nine B-ALL PDX having only gone through a single round of amplification in mice previous to these tests. All xenograft samples showed high sensitivity to RSL3 treatment and were even more sensitive than the positive control 
cell lines, NALM6 (Fig. 5A). Furthermore, ferrostatin-1 treatment rescued RSL3 sensitivity in all

206 PDX, indicating that ferroptosis is the major cell death mechanism in these RSL3-treated PDX. B-

207 ALL PDX were also sensitive to three other ferroptosis-inducing drugs - erastin, FIN56 and

208 sulfasalazine - although all PDX were not necessarily sensitive to all drugs (Fig. 5B-D). Erastin

209 and sulfasalazine demonstrated a similar sensitivity profile consistent with these two drugs

210 targeting the $\mathrm{Xc}^{-}$transporter. In all, these experiments confirm the extreme sensitivity of B-ALL

211 cell lines and patient-derived samples to ferroptosis induction.

\section{Discussion}

213 Despite the continuous improvement in treatment outcome and the greater understanding of the 214 molecular pathogenesis underlying tumor development, B-ALL still poses unresolved clinical 215 needs, especially to relapsed patient. Developing additional therapeutic avenues by identifying 216 new vulnerabilities is therefore critical to provide new strategies for cancer treatment. In an attempt 217 to fulfill this gap, we performed whole-genome pooled dropout CRISPR screens in B-ALL cell 218 lines. These screens found essential genes that were mostly shared with other cancer histotypes; 219 these genes were enriched in core essential genes mostly implicated in general processes such as 220 transcription, translation, proteasome, etc. In addition, these screens also identified a panel of more 221 than three hundred genes that were essential in most ALL cell line, but not across other tumor 222 histotypes $^{7}$, suggesting that these genes represent unique functional vulnerabilities to B-ALL. 223 These were enriched for well-described B-ALL specific transcription factors (PAX5, RUNX1, $224 T C F 3, E B F 1)$ and signal transduction pathways (CD79A, CD79B, PIK3CD), but also novel 225 vulnerabilities not previously linked to B-ALL, several of which directly regulate ferroptosis 226 induction.

227 Ferroptosis is a recently described iron-dependent cell death pathway that is characterized by the 228 excessive peroxidation of phospholipid at the cell membranes. This non-apoptotic cell death 229 pathway has garnered increasing interest as a potential novel cancer therapy since "persister" cells 230 rendered drug-tolerant through serial exposure to chemotherapeutic agents and cells that 231 underwent an epithelial-mesenchymal transition, two states associated with resistance to cancer 232 therapy, demonstrate exquisite sensitivity to ferroptosis induction ${ }^{27,34}$. The main gatekeeper for 233 ferroptosis induction is GPX4, a glutathione peroxidase, which uses GSH as an obligate co-factor 
and possess the unique ability to detoxify hydroxyperoxides in complex lipids. Our findings add

235 B-ALL lines to the cell lines or cell state that have been reported to be sensitive to GPX4 inhibition by either ferroptosis inducing drug ${ }^{12,26,27}$ or direct gene knock-out ${ }^{35}$. To our knowledge, this is the

237 first evidence of such a sensitivity in these cells and the first demonstration that this sensitivity can

238 be exploited therapeutically (see below). Many genes modulating ferroptosis induction are still not

239 labelled as such by KEGG or MSigDB enrichment tools ${ }^{36-38}$, especially for genes involved in the

240 multiple metabolic pathways that are indirectly regulating GPX4 activity (Fig. 6). This explains

241 why ferroptosis pathway, even if significantly enriched, was not ranked higher in our Wikipathway

242 analysis (Supplemental Table 5) even though many B-ALL essential genes were involved in

243 pathways related to GPX4 activity, such as selenocompound, lipid, mevalonate, GSH and iron

244 metabolism (Fig. 6). We validated several of these genetic dependencies using orthogonal assays

245 and demonstrated that inhibition of these pathways induce ferroptosis in B-ALL cells. As shown

246 by our +/- ferrostatin-1 screens, apart from GPX4, B-ALL cells were also particularly sensitive to

247 the depletion of genes implicated in selenocysteine synthesis (Fig. 3H). Selenocysteine

248 incorporation into GPX4 is required for its ferroptosis inhibitor activity ${ }^{32}$ and co-essentiality of

249 selenocysteine synthesis genes with GPX4 was previously demonstrated in glioblastoma cells ${ }^{39}$.

250 These results also suggest that the primary role of selenocysteine metabolism in ALL cells is to

251 synthesize GPX4 as none of the 24 other selenocysteine-containing proteins were essential in our

252 screen. This is reminiscent of genetic deletion of selenocysteine-containing proteins in mice where

$253 \mathrm{KO}$ of GPX4 is the only one that is embryonic lethal, a phenotype shared with selenocysteine

254 tRNA KO mice ${ }^{40,41}$.

255 Several elements seem to synergize to explain the high sensitivity of B-ALL cells to ferroptosis 256 induction. High lipid ROS levels in steady-state conditions suggest that B-ALL are under constant 257 oxidative stress. Combined with the low GSH and FSP1 antioxidant levels, it implies that these 258 cells do not possess the buffering capacity that would normally protect them against oxidative 259 stress. And while restoring FSP1 expression levels in B-ALL cell increases resistance to 260 ferroptosis induction (Fig. 4C), this only partially rescues sensitivity compared to other resistant 261 cell lines, suggesting that additional elements contribute to the extreme sensitivity of B-ALL cells 262 to ferroptosis induction. One explanation might lie in the high levels of PUFA that were measured 263 in B-ALL ${ }^{42}$ since a direct correlation between PUFA levels in cell lines and GPX4 KO sensitivity 264 has been established by metabolite dependency association studies ${ }^{43}$. In addition, transcriptional 
repression of the pentose phosphate pathway (PPP) by B cell-specific transcriptional factor PAX5 and IKZF1 in B-ALL limits its activity and ability to cope with oxidative stress ${ }^{44}$. This particular vulnerability results in low levels of NADPH, which prevents GSSG reduction into GSH. The limited PPP activity appears to be controlled both by the serine-threonine protein phosphatase $2 \mathrm{~A}$ (PP2A) and the Cyclin D3-CDK6 kinase; inhibition of the latter reduces the flow of carbon through the PPP in favor of glycolysis ${ }^{45}$. Cyclin D3-CDK6 kinase regulates the switch from glycolysis to the PPP by directly phosphorylating and inhibiting key enzymes, which catalyse key rate-limiting steps in the glycolysis cascade such as 6-phosphofructokinase (PFK1), pyruvate kinase M2 (PKM2), but also GPI, PGK1, ENO1, and PKM. Notably, the last four enzymes along with Cyclin D3 (CCND3) and CDK6 are all considered "B-ALL enriched essential genes" according to our screens. Overall, these results, along with the one presented in this paper, suggest that the low PPP activity found in B-ALL, which leads to low levels of NADPH and GSH, restricts their ability to cope with oxidative stress. This strenuous balance can be easily tipped toward ferroptosis induction when GPX4 is inhibited.

Most conventional chemotherapeutic agents currently use for treating ALL patients were approved more than 40 years ago and dosage and schedule have been fully optimized such that modifying the current treatment regimen will purportedly minimally improve outcome ${ }^{46}$. Recently, molecular 282 targeted therapies such as BCR-ABL, JAK, mTOR, and proteasome inhibitors and immunotherapies such as monoclonal antibodies and CAR-T cells have shown great promises for improving outcomes ${ }^{47}$. However, the number of individuals benefiting from these novel therapies remain low for several reasons (expression of the target, resistance, toxicity, etc) such that both novel targets and therapeutics are needed to achieve greater clinical success. Ferroptosis sensitivity was seen across multiple B-ALL subtypes since several cell lines and PDX were sensitive to drugs inducing ferroptosis (Fig. 2, 3, 5 and 6). Because B-ALL are primed to undergo ferroptosis and multiple metabolic pathways modulate GPX4 activity, several therapeutic opportunities are potentially available to target this vulnerability. As shown by our results and others, ALL cells are sensitive to FDA approved drug such as sulfasalazine (Fig. 5D), BSO (Fig. 3A), and statins (Fig.

$2923 \mathrm{E}$ and ${ }^{48}$ ). Since many chemotherapies have been shown to induce oxidative stress in cancer cells, 293 combining these treatments with a ferroptosis inducing agent could help prevent the development

294 of treatment-resistant tumor cells. Finally, according to gene/protein expression analyses, other 295 leukemias, such as T-ALL and AML, also share similar expression profile for gene controlling 
sensitivity to ferroptosis induction and would also likely benefit from therapeutic strategies develop for ALL. Overall, our work identified a comprehensive set of genetic dependencies and molecular mechanisms sustaining tumorigenesis in B-ALL, some of which can be further exploited therapeutically.

\section{Methods}

\section{Cell lines and Cell Culture}

B-ALL cell lines used for this study were NALM6, HAL-01, REH, 697, RS4;11, RCH-ACV,

303 Tanoue, SEM (DSMZ, Germany). A549 (lung carcinoma), MCF7 (breast adenocarcinoma) and 304 NCI-H226 (lung squamous cell carcinoma) were obtained from ATCC. Cell lines were cultured 305 in RPMI 1640 media $\left(\right.$ HyClone $\left.^{\mathrm{TM}}\right)+10 \%$ heat-inactivated FBS $\left(\mathrm{Gibco}^{\mathrm{TM}}\right)+2 \mathrm{mM}$ glutamine $306\left(\mathrm{Gibco}^{\mathrm{TM}}\right)$ at $37^{\circ} \mathrm{C}$ in $5 \% \mathrm{CO}_{2}$ incubators. HEK293SF-3F6 (ATCC) were grown in HyCell 307 TransFx-H media (Hyclone ${ }^{\mathrm{TM}}$, Fisher Scientific) $+4 \mathrm{mM}$ L-glutamine $\left(\right.$ HyClone $\left.^{\mathrm{TM}}\right)+0,1 \%$ 308 Kolliphor (Sigma) in shaker flasks, at $120 \mathrm{rpm}, 37^{\circ} \mathrm{C}$ in $5 \% \mathrm{CO}_{2}$ incubators.

\section{B-ALL screens}

B-ALL cells were infected with Cas9-2A-blast lentivirus followed by one-week selection with 5-15 $\mu \mathrm{g} / \mathrm{mL}$ blasticidin (InvivoGen, CA). Cells were amplified and tested for Cas9 activity using

312 the reporter assay described in Tzelepis et al. ${ }^{14}$ Pools with $>75 \%$ Cas 9 activity were used going 313 forward. Cas9-expressing B-cell stable pools were transduced at 0.3 multiplicity of infection 314 (M.O.I). with the pLKV2 whole genome human library, at 300-fold representation in triplicate 315 ( 30 million cells per replicate). 24h post-infection, cells were centrifuged and resuspended in 316 fresh media + 0.5-7.5 $\mu \mathrm{g} / \mathrm{mL}$ puromycin $\left(\mathrm{Gibco}^{\mathrm{TM}}\right) .48 \mathrm{~h}$ post puromycin addition, cells were 317 counted with Vi-Cell ${ }^{\mathrm{TM}}$ XR (Beckman Coulter, IN) using Trypan Blue for cell viability assessment 318 and 30 million cells were pelleted per replicate for T0 time points. M.O.I. evaluation was done 319 using CellTiter-Glo® (Promega, WI) and by comparing luminescence of infected cells without 320 puromycin selection and luminescence of cells under puromycin selection. Luminescence was 321 measured using a Synergy 2 luminometer (BioTek $\left.{ }^{\circledR}\right)$ in 96-well white plates. 30 million cells per 322 replicate were plated in culture and left for about 14 doublings with regular passage (2-4 days). 323 After 14 doublings, 30 million cells per replicate were pelleted for Tf time points. 


\section{Gene network analysis}

325 Key pathways associated with ALL specific genes were determined by mapping ALL enriched essential genes onto the STRING protein database ${ }^{49}$. High confidence protein-protein interactions

327 were retained (score $>0.7$ ). We used Cytoscape and maximum clique centrality (MCC) to identify 328 the top 100 hub genes ${ }^{50,51}$. For each hub (cluster), a KEGG analysis was performed to highlight 329 key pathways in the network ${ }^{38}$.

\section{Drug treatments}

331 Ferroptosis-modulating drugs were procured from Millipore Sigma (Oakville, ON, Canada). 332 DMSO was from Santa Cruz Biotechnologies (Dallas, TX). IC50 curves and drug rescue were 333 measured using CellTiter-Glo® (Promega, WI) and luminescence was measured as described 334 above. For drug rescue experiments, both drugs were added simultaneously.

\section{Flow Cytometry analysis}

336 Cells treated with $1 \mu \mathrm{M}$ RSL3 for 4 h were stained with $200 \mu \mathrm{M}$ C11 581/591 BODIPYTM or 337 Annexin V Pacific Blue ${ }^{\mathrm{TM}}$ conjugate (Molecular Probes ${ }^{\circledR}$ ) as per manufacturer protocol. A 20h 8 $338 \mu \mathrm{M}$ etoposide treatment was used as a control for apoptosis induction. For each condition, 339 approximately 30,000 cells were analyzed by flow cytometry with a BD LSRFortessa ${ }^{\mathrm{TM}}$ system 340 (BD Biosciences, CA). Data were analyzed using the BD FACSDiva ${ }^{\mathrm{TM}}$ Software (BD 341 Biosciences).

\section{Screen +/- Ferrostatin}

343 REH and SEM Cas9 pools were screened as described above, except that each pool was screened $344+/-4 \mu \mathrm{M}$ ferrostatin in parallel. Ferrostatin and puromycin were added $24 \mathrm{~h}$ post transfection. 345 Sequence analysis was performed using MAGeCK Maximum Likelihood Estimation (MLE) 346 algorithm with combined data from two cell lines and the MAGeCKFlute R package (version 347 1.4.3) used to highlight treatment specific genes ${ }^{52}$. 
Lentiviruses expressing FSP1-myc-DDK-P2A-puro were used to infect NALM6 and REH cell

350 lines. FSP1 expression levels were validated by WB. Following puromycin selection, pools were

351 used in parallel with parental cells to perform RSL3 IC50 curves as described above.

\section{Patient-derived xenografts and drug treatments}

353 PDX were generated as described in details in Nicolas Montpas et al. (manuscript in preparation).

354 Briefly, bone marrow samples of B-ALL patients were collected at diagnosis and cells were

355 isolated by Ficoll-Paque. 0.6-5 × $10^{6}$ cells were injected in the tail vein of NOD-scidILR2 gamma ${ }^{\text {null }}$

356 (NSG) mice and blast percentage (blast\%) in the blood was monitored by monthly bleeding using

357 human CD45, CD10 and CD19 vs CD45 murine antibodies (Biolegend, CA). Cells were expanded

358 until blast $\%>1$. Mice were then sacrificed, and PDX were isolated by extracting pre-B cells from

359 mice spleen. Drug treatments were done for $36 \mathrm{~h}$ before cell viability assessment by CellTiter-Glo ${ }^{\circledR}$

360 (Promega, WI). Detailed information on each PDX can be found in Suppl. Table 7.

\section{Acknowledgments}

362 We thank Sonia Leclerc for performing Next-Generation Sequencing experiments. We also thank

363 Nadine Fradet and Mylène Gosselin for their contribution to early development of the experimental

364 designs. All authors are members of the NRC-CHUSJ Collaborative Unit for Translational

365 Research (CUTR). We would like to acknowledge CUTR for funding part of this work. This is

366 NRC publication \# 53536.

\section{Author Contributions}

368 Contribution: MEL and RM designed the study and prepared the manuscript; MEL and AMG 369 performed the experiments; MEL, MS, AMG, JSM and RM analyzed the data; KB and EH 370 provided patient samples; and all authors edited and approved the manuscript.

\section{Competing Interests}

372 The authors declare no competing financial interests. 
375

376

1. Sabattini, E., Bacci, F., Sagramoso, C. \& Pileri, S. A. WHO classification of tumours of haematopoietic and lymphoid tissues in 2008: an overview. Pathologica 102, 83-87 (2010).

2. Ellison, L. F., De, P., Mery, L. S., Grundy, P. E., \& for the Canadian Cancer Society's Steering Committee for Canadian Cancer Statistics. Canadian cancer statistics at a glance: cancer in children. Can. Med. Assoc. J. 180, 422-424 (2009).

3. Vrooman, L. M. \& Silverman, L. B. Treatment of Childhood Acute Lymphoblastic Leukemia: Prognostic Factors and Clinical Advances. Curr. Hematol. Malig. Rep. 11, 385394 (2016).

4. Bruzzi, P. et al. Long-term effects on growth, development, and metabolism of ALL treatment in childhood. Expert Rev. Endocrinol. Metab. 14, 49-61 (2019).

5. Meyers, R. M. et al. Computational correction of copy number effect improves specificity of CRISPR-Cas9 essentiality screens in cancer cells. Nat. Genet. 49, 1779-1784 (2017).

6. Behan, F. M. et al. Prioritization of cancer therapeutic targets using CRISPR-Cas9 screens. Nature 568, 511-516 (2019).

7. Dempster, J. M. et al. Agreement between two large pan-cancer CRISPR-Cas9 gene dependency data sets. Nat. Commun. 10, 5817 (2019).

8. Hart, T. et al. High-Resolution CRISPR Screens Reveal Fitness Genes and GenotypeSpecific Cancer Liabilities. Cell 163, 1515-1526 (2015).

9. Hassannia, B., Vandenabeele, P. \& Vanden Berghe, T. Targeting Ferroptosis to Iron Out Cancer. Cancer Cell 35, 830-849 (2019).

10. Jiang, X., Stockwell, B. R. \& Conrad, M. Ferroptosis: mechanisms, biology and role in disease. Nat. Rev. Mol. Cell Biol. 22, 266-282 (2021).

11. Wang, L., Chen, X. \& Yan, C. Ferroptosis: An emerging therapeutic opportunity for cancer. Genes Dis. S2352304220301239 (2020) doi:10.1016/j.gendis.2020.09.005.

12. Doll, S. et al. FSP1 is a glutathione-independent ferroptosis suppressor. Nature 575, 693698 (2019).

13. Bersuker, K. et al. The CoQ oxidoreductase FSP1 acts parallel to GPX4 to inhibit ferroptosis. Nature 575, 688-692 (2019).

14. Tzelepis, K. et al. A CRISPR Dropout Screen Identifies Genetic Vulnerabilities and Therapeutic Targets in Acute Myeloid Leukemia. Cell Rep. 17, 1193-1205 (2016).

15. Hart, T. \& Moffat, J. BAGEL: a computational framework for identifying essential genes from pooled library screens. BMC Bioinformatics 17, 164 (2016).

16. Metzeler, K. H. \& Bloomfield, C. D. Clinical Relevance of RUNX1 and CBFB Alterations in Acute Myeloid Leukemia and Other Hematological Disorders. Adv. Exp. Med. Biol. 962, 175-199 (2017).

17. Gu, Z. et al. PAX5-driven subtypes of B-progenitor acute lymphoblastic leukemia. Nat. Genet. 51, 296-307 (2019).

18. Hein, D., Borkhardt, A. \& Fischer, U. Insights into the prenatal origin of childhood acute lymphoblastic leukemia. Cancer Metastasis Rev. 39, 161-171 (2020).

19. Chu, P. G. \& Arber, D. A. CD79: a review. Appl. Immunohistochem. Mol. Morphol. AIMM 9, 97-106 (2001).

20. Kruth, K. A. et al. Suppression of B-cell development genes is key to glucocorticoid efficacy in treatment of acute lymphoblastic leukemia. Blood 129, 3000-3008 (2017).

21. Serafin, V. et al. SYK Targeting Represents a Potential Therapeutic Option for Relapsed Resistant Pediatric ETV6-RUNX1 B-Acute Lymphoblastic Leukemia Patients. Int. J. Mol. Sci. 20, (2019). 
421

422

423

424

425

426

427

428

429

430

431

432

433

434

435

436

437

438

439

440

441

442

443

444

445

446

447

448

449

450

451

452

453

454

455

456

457

458

459

460

461

462

463

464

465

466
22. Bortolozzi, R. et al. Ribociclib, a Cdk4/Cdk6 kinase inhibitor, enhances glucocorticoid sensitivity in B-acute lymphoblastic leukemia (B-All). Biochem. Pharmacol. 153, 230-241 (2018).

23. Ott, C. J. et al. BET bromodomain inhibition targets both c-Myc and IL7R in high-risk acute lymphoblastic leukemia. Blood 120, 2843-2852 (2012).

24. Scheffold, A., Jebaraj, B. M. C. \& Stilgenbauer, S. Venetoclax: Targeting BCL2 in Hematological Cancers. Recent Results Cancer Res. Fortschritte Krebsforsch. Progres Dans Rech. Sur Cancer 212, 215-242 (2018).

25. Dixon, S. J. et al. Ferroptosis: An Iron-Dependent Form of Nonapoptotic Cell Death. Cell 149, 1060-1072 (2012).

26. Yang, W. S. et al. Regulation of Ferroptotic Cancer Cell Death by GPX4. Cell 156, 317-331 (2014).

27. Hangauer, M. J. et al. Drug-tolerant persister cancer cells are vulnerable to GPX4 inhibition. Nature 551, 247-250 (2017).

28. Zhang, Y. et al. mTORC1 couples cyst(e)ine availability with GPX4 protein synthesis and ferroptosis regulation. Nat. Commun. 12, 1589 (2021).

29. Wang, F. et al. Iron and leukemia: new insights for future treatments. J. Exp. Clin. Cancer Res. 38, 406 (2019).

30. Griffith, O. W. Mechanism of action, metabolism, and toxicity of buthionine sulfoximine and its higher homologs, potent inhibitors of glutathione synthesis. J. Biol. Chem. 257, 13704-13712 (1982).

31. Moosmann, B. \& Behl, C. Selenoproteins, Cholesterol-Lowering Drugs, and the Consequences Revisiting of the Mevalonate Pathway. Trends Cardiovasc. Med. 14, 273-281 (2004).

32. Ingold, I. et al. Selenium Utilization by GPX4 Is Required to Prevent HydroperoxideInduced Ferroptosis. Cell 172, 409-422.e21 (2018).

33. Shimada, K. et al. Global survey of cell death mechanisms reveals metabolic regulation of ferroptosis. Nat. Chem. Biol. 12, 497-503 (2016).

34. Viswanathan, V. S. et al. Dependency of a therapy-resistant state of cancer cells on a lipid peroxidase pathway. Nature 547, 453-457 (2017).

35. Tsherniak, A. et al. Defining a Cancer Dependency Map. Cell 170, 564-576.e16 (2017).

36. Subramanian, A. et al. Gene set enrichment analysis: a knowledge-based approach for interpreting genome-wide expression profiles. Proc. Natl. Acad. Sci. U. S. A. 102, 1554515550 (2005).

37. Liberzon, A. et al. The Molecular Signatures Database (MSigDB) hallmark gene set collection. Cell Syst. 1, 417-425 (2015).

38. Kanehisa, M. \& Goto, S. KEGG: kyoto encyclopedia of genes and genomes. Nucleic Acids Res. 28, 27-30 (2000).

39. Kim, E. et al. A network of human functional gene interactions from knockout fitness screens in cancer cells. Life Sci. Alliance 2, (2019).

40. Ufer, C. \& Wang, C. C. The Roles of Glutathione Peroxidases during Embryo Development. Front. Mol. Neurosci. 4, 12 (2011).

41. Yant, L. J. et al. The selenoprotein GPX4 is essential for mouse development and protects from radiation and oxidative damage insults. Free Radic. Biol. Med. 34, 496-502 (2003).

42. Agatha, G., Häfer, R. \& Zintl, F. Fatty acid composition of lymphocyte membrane phospholipids in children with acute leukemia. Cancer Lett. 173, 139-144 (2001). 
43. Li, H. et al. The landscape of cancer cell line metabolism. Nat. Med. 25, 850-860 (2019).

44. Xiao, G. et al. B-Cell-Specific Diversion of Glucose Carbon Utilization Reveals a Unique Vulnerability in B Cell Malignancies. Cell 173, 470-484.e18 (2018).

45. Wang, H. et al. The metabolic function of cyclin D3-CDK6 kinase in cancer cell survival. Nature 546, 426-430 (2017).

46. Inaba, H. \& Pui, C.-H. Advances in the Diagnosis and Treatment of Pediatric Acute Lymphoblastic Leukemia. J. Clin. Med. 10, 1926 (2021).

47. Annesley, C. E. \& Brown, P. Novel agents for the treatment of childhood acute leukemia. Ther. Adv. Hematol. 6, 61-79 (2015).

48. Sheen, C. et al. Statins are active in acute lymphoblastic leukaemia (ALL): a therapy that may treat ALL and prevent avascular necrosis. Br. J. Haematol. 155, 403-407 (2011).

49. Szklarczyk, D. et al. STRING v11: protein-protein association networks with increased coverage, supporting functional discovery in genome-wide experimental datasets. Nucleic Acids Res. 47, D607-D613 (2019).

50. Shannon, P. et al. Cytoscape: a software environment for integrated models of biomolecular interaction networks. Genome Res. 13, 2498-2504 (2003).

51. Chin, C.-H. et al. cytoHubba: identifying hub objects and sub-networks from complex interactome. BMC Syst. Biol. 8 Suppl 4, S11 (2014).

52. Wang, B. et al. Integrative analysis of pooled CRISPR genetic screens using MAGeCKFlute. Nat. Protoc. 14, 756-780 (2019).

\section{Figure Legends}

\section{Figure 1. Whole-genome CRISPR/Cas9 screens of ALL cell lines identify ALL essential} genes. A) Schema of B-ALL screening pipeline B) Significant genes set intersection of 7 B-ALL cell line CRISPR screen results with UpSet, "Broad” and "Sanger”(Dempster et al. 2019) essential gene list at false discovery rate $(\mathrm{FDR})<0,05$. The intersections with more than 5 genes are shown. C) Overlap between B-ALL essential ( $>4 / 7$ cell lines, FDR <0.05) genes, "Broad" and "Sanger" core essential genes. D) Volcano plot of 697 cells CRISPR screen results with FDR $<0.05$. B-ALLknown dependencies are highlighted in blue and ALL enriched essential genes are highlighted in light blue. Volcano plot of 697 cells is shown as a representative Volcano plot of B-ALL cells screen results. E) Gene Network Analysis of B-ALL enriched essential genes where nodes represent genes and edges represent protein-protein interactions between genes. Node color represents the number of ALL cell lines essential for the given gene. F) Log2 fold change distribution of sgRNAs in the 7 ALL screened cell lines. sgRNAs Log2 fold change for ferroptosis 
502

503

504

505

506

507

508

509

510

511

512

513

514

515

516

517

518

519

520

521

522

523

524

525

526

527

528

529

530

related genes are illustrated at bottom of the panel. VAX1, negative control. TP53 was enriched in several B-ALL cell lines.

Figure 2. B-ALL are highly sensitive to GPX4 inhibition. A) Cell viability curves of B-ALL and other cell lines (A549, MCF7, NCI-H226) to the GPX4 inhibitor (RSL3). B) Growth of REH GFP clones 1 and 2 (negative control) and REH GPX4 KO clones 2 and $6+/-$ ferrostatin $(2 \mu \mathrm{M})$ for 18h. C) WB analysis of GPX4 protein level in GPX4 KO vs GFP KO clones. D) RSL3 (1 $\mu \mathrm{M})$ rescue experiments with the iron chelator deferoxamine (DFO; $10 \mu \mathrm{M})$ ), the pan-caspase apoptotic inhibitor ZVADK (50 $\mu \mathrm{M})$, the 15-lipoxygenase-1 inhibitor PD146176 (0.5 $\mu \mathrm{M})$, the superoxide dismutase mimetic EUK-134 $(30 \mu \mathrm{M})$, or the radical trapping agent ferrostatin $(2 \mu \mathrm{M})$ in NALM6 and RS4;11 for $24 \mathrm{~h} . * *$ Unpaired t-test pvalue $<0,001$ and $* * *$ pvalue $<0,0001$.

Figure 3. Pathways controlling GPX4 activity contribute to ALL sensitivity. A) WB antiSLC7A11 in B ALL cell lines. $\beta$-actin is used as loading control B) GSH level measurement in steady-state conditions for ALL vs non-GPX4 sensitive (non-ALL) cells. ***Unpaired t-test pvalue $<0,0001$. C) IC50 curves of BSO in ALL cell lines vs non-GPX4 sensitive (non-ALL) cell lines after $96 \mathrm{~h}$ treatment. Data shown are from one representative experiment. D) IC50 curves of Erastin inhibitor in ALL vs non-GPX4 sensitive (non-ALL) cells. Data shown are from one representative experiment. E) $10 \mathrm{uM}$ erastin- (system Xc- transporter inhibitor) and $100 \mathrm{uM} \mathrm{BSO}$ treated NALM6 and RS4;11 are rescued by 1mM GSH (48h). *** Unpaired t-test pvalue $<0,001$ and ** pvalue $<0,01$. F) IC50 curves of Fin56 inhibitor in ALL vs non-GPX4 sensitive (non-ALL) cells. Data shown are from one representative experiment. G) Cell viability of NALM6 and RS4;11 following treatment with lovastatin $(20 \mathrm{uM})$ and simvastatin $(20 \mathrm{uM})$ for $72 \mathrm{~h}$. *** Unpaired t-test pvalue $<0,002$ and $* *$ pvalue $<0,01 . \mathrm{H}$ ) Beta scores for $+/$ - ferrostatin rescue screens performed in $\mathrm{REH}^{\mathrm{cas} 9}$ and SEM ${ }^{\mathrm{cas} 9}$ pools. Only GPX4 and selenocompound metabolism genes are indicated. Values represent $\log 2$ fold-change between untreated (control) and treated samples.

Figure 4. Low level of FSP1 in B-ALL contributes to ferroptosis sensitivity. A) FSP1 protein level comparison between GPX4 sensitive and non-sensitive CCLE cell lines by cancer type. (see material and method) B) FSP1 WB on parental vs FSP1 overexpressing B-ALL cells. B-actin is shown as a loading control. C) RSL3 dose-response curves in B-ALL pools overexpressing high or low levels of FPS1. 
531 Figure 5. B-ALL patient derived xenografts are sensitive to ferroptosis inducing drugs. B-

532 ALL patient-derived xenografts were treated with A) RSL3 +/- ferrostatin, B) erastin, C) Fin56,

533 and D) sulfasalazine. Cell viability was assessed $36 \mathrm{~h}$ post-treatment and compared to untreated

534 xenografts.

535 Figure 6. Integration of GPX4-related pathways. Several genes/pathways found essential in

536 ALL cells potentially regulate GPX4 activity which may explain their acute vulnerability to

537 ferroptosis induction. Genes in bold characters are found within the 319 ALL enriched essential 538 genes. 
bioRxiv preprint doi: https://doi.org/10.1101/2022.02.01.478478; this version posted February 1,2022. The copyright holder for this preprint (which was not certified by peer review) is the author/funder, who has granted bioRxiv a license to display the preprint in perpetuity. It is made

A Cas9-2A-blast lentivirus ALL cell lines astiailable under aCC-BY-NC-ND 4.0 International license. C

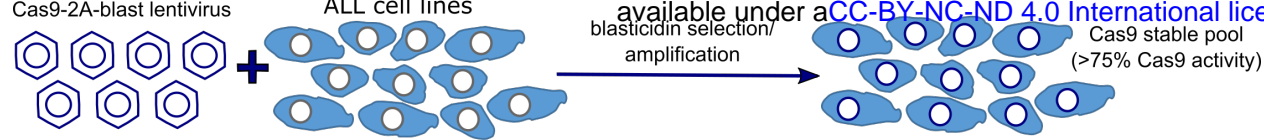

(a) (O)

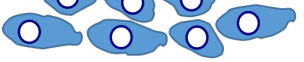

Initial (T0) pool of cells
1 sgRNA/cell

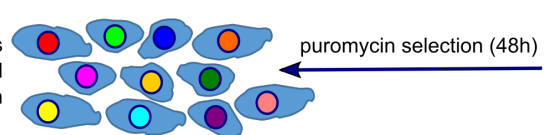

$+$ $300 \mathrm{X}$ representatio

(a) (1) (1) whole-genome (O) (0) (0) $0.3 \mathrm{~mol}$
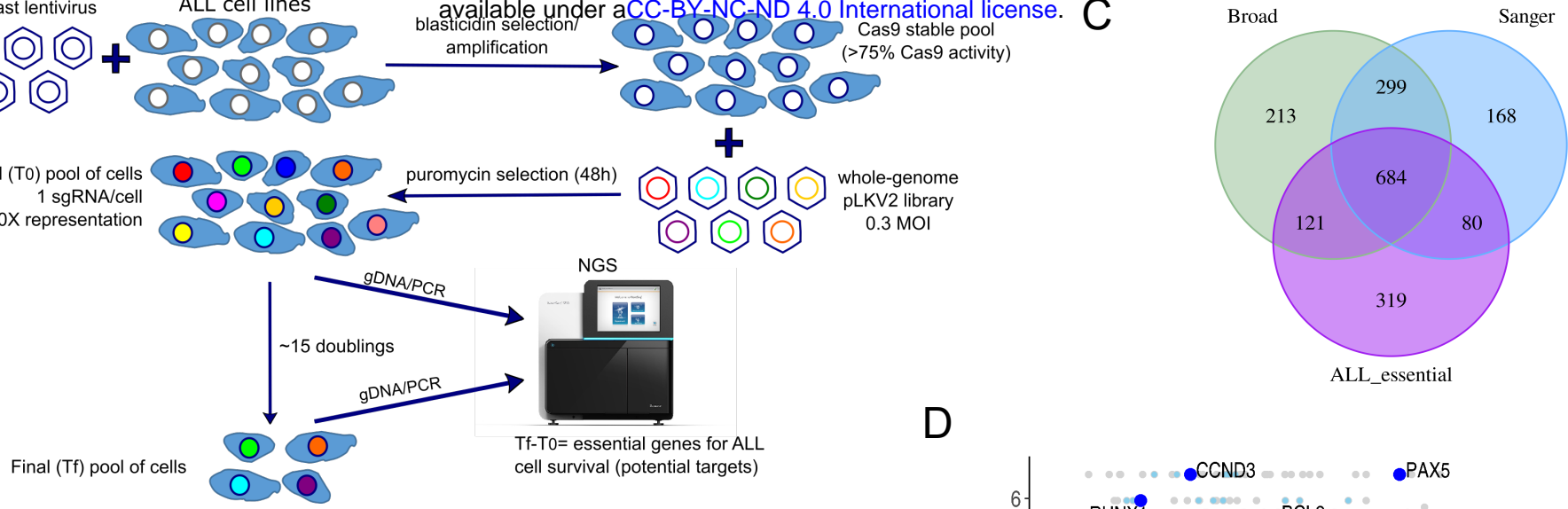

ALL_essential

D

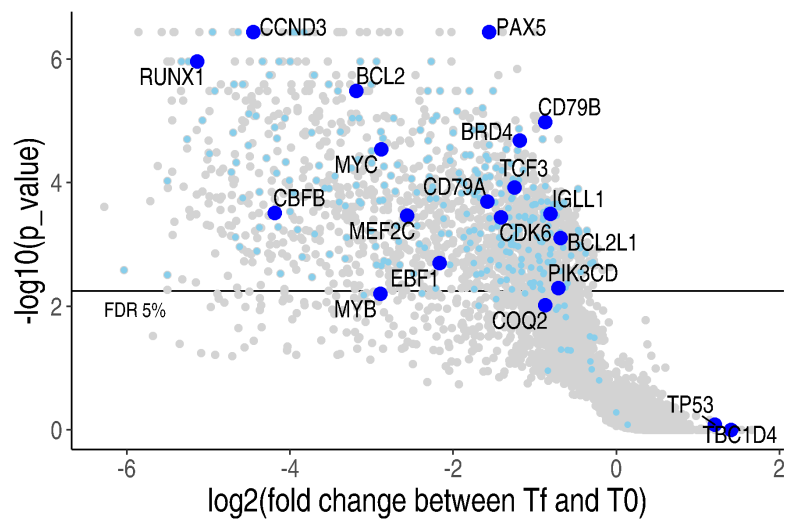

B
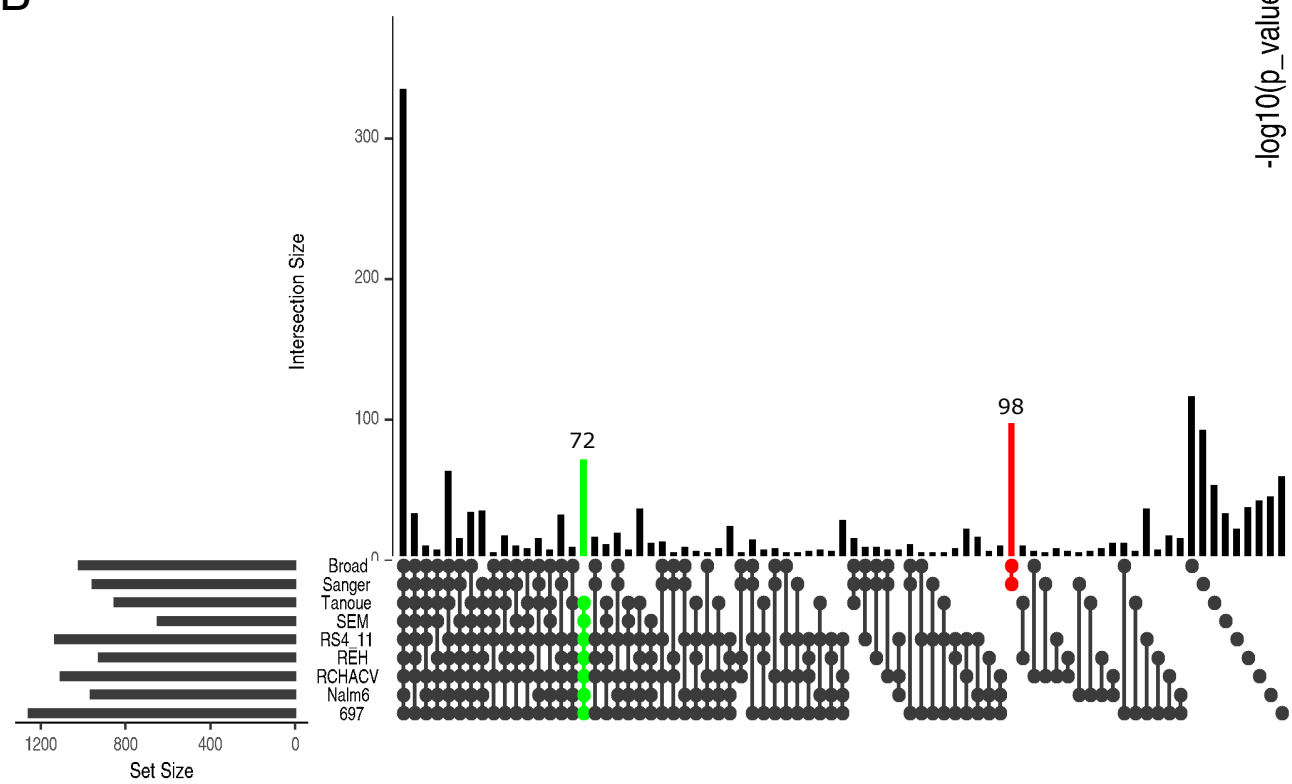

$E$

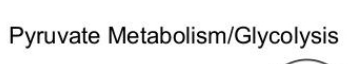

Mitochondrial Translation
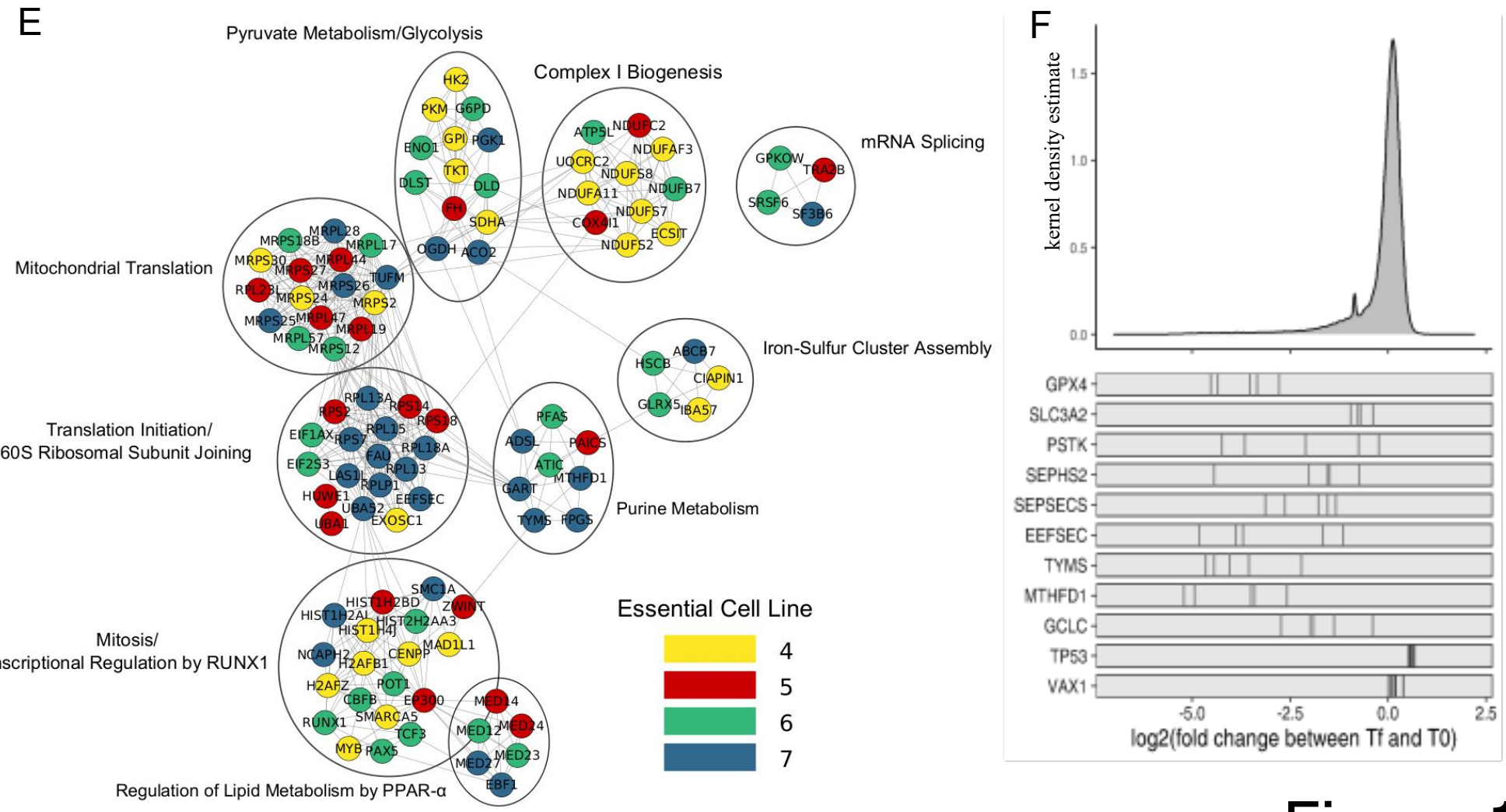
bioRxiv preprint doi: https://doi.org/10.1101/2022.02.01.478478 this version posted February 1 , 2022. The copyright holder for this preprint A (which was not certified by peer review) is the author/funder, whd has granted bioRxiv a license to display the prepsint in perpetuity. It is made

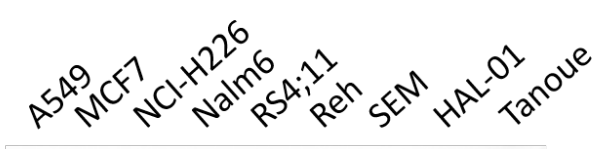

- - Anti-XCT (SLC7A11)

Anti-B-actin

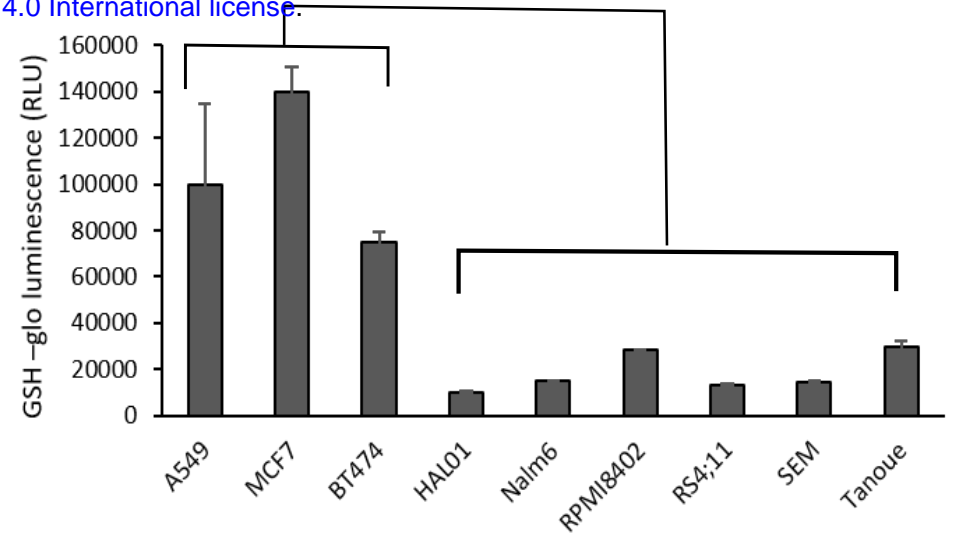

C

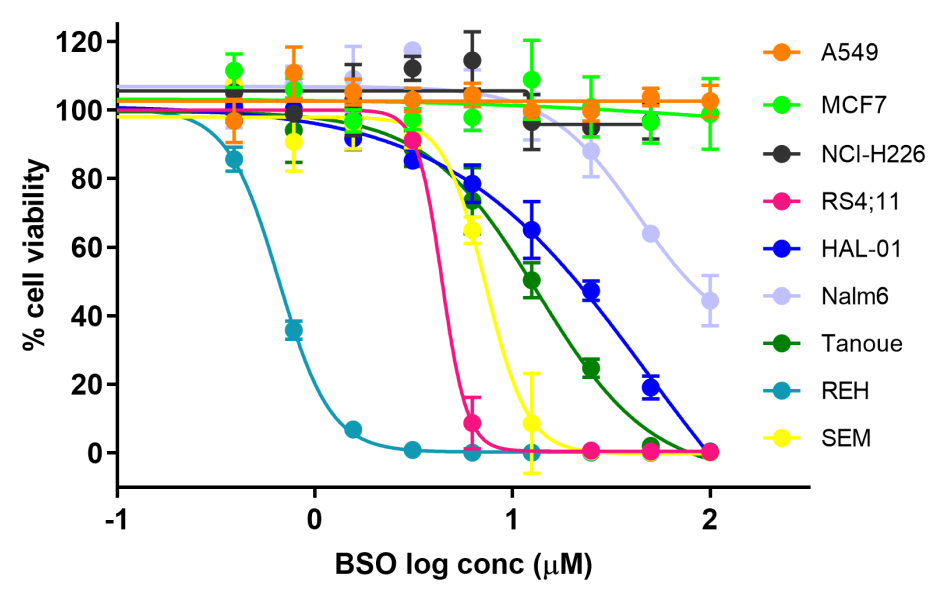

E

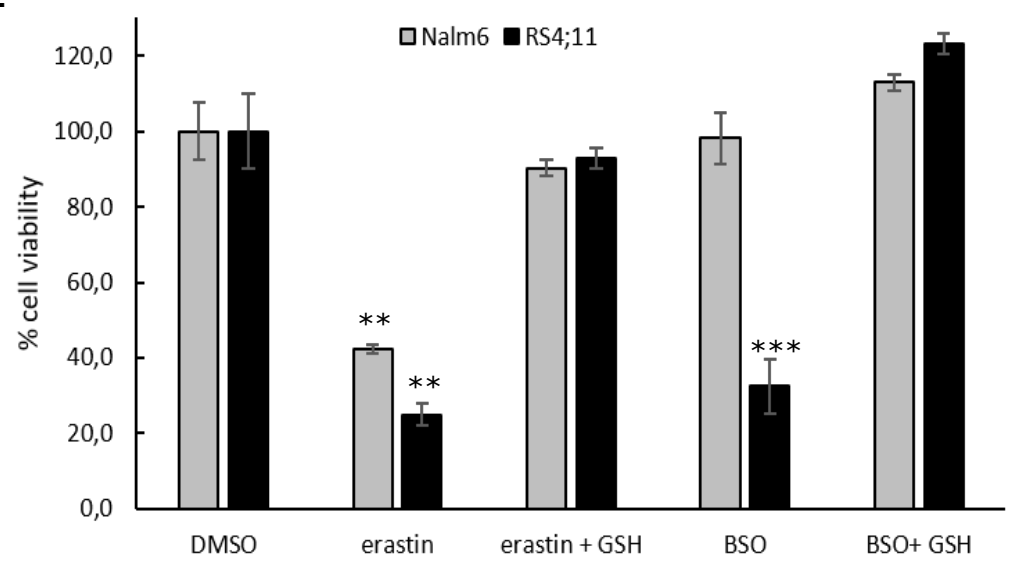

G

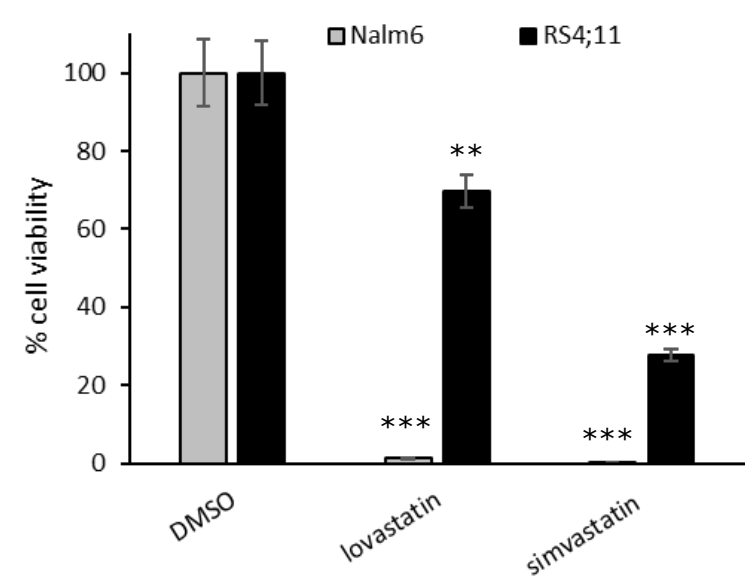

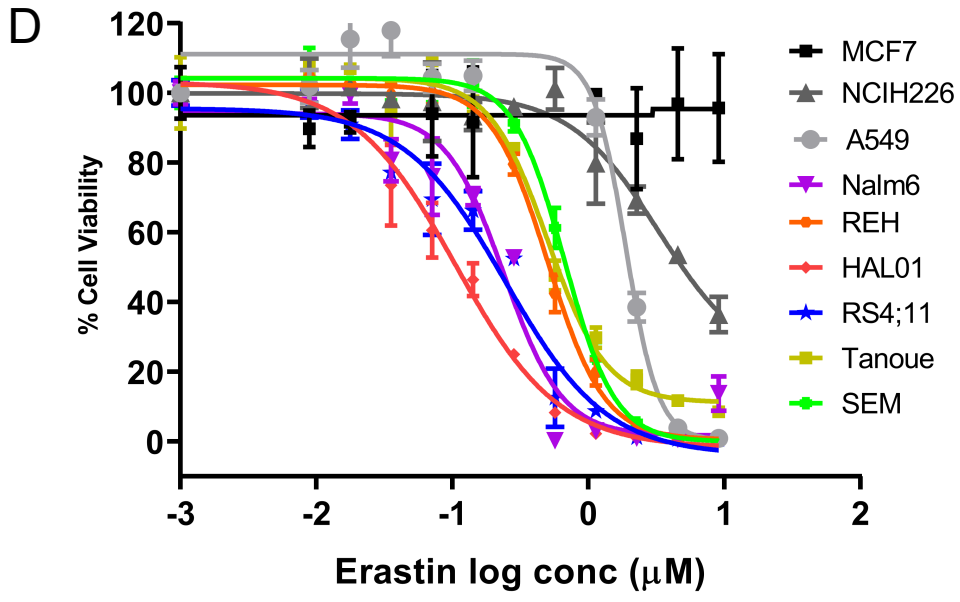

F

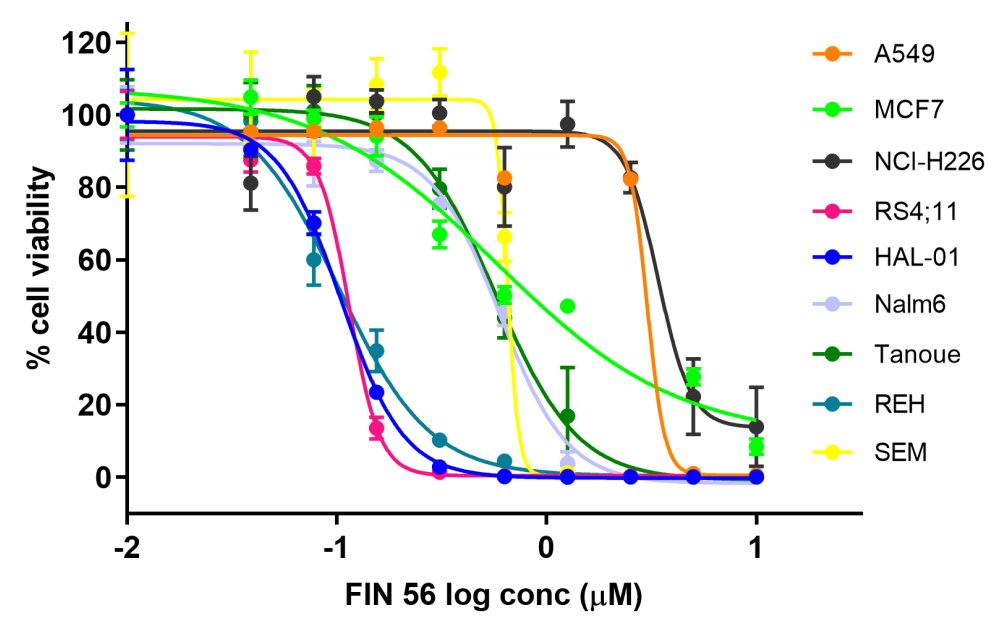

$\mathrm{H}$

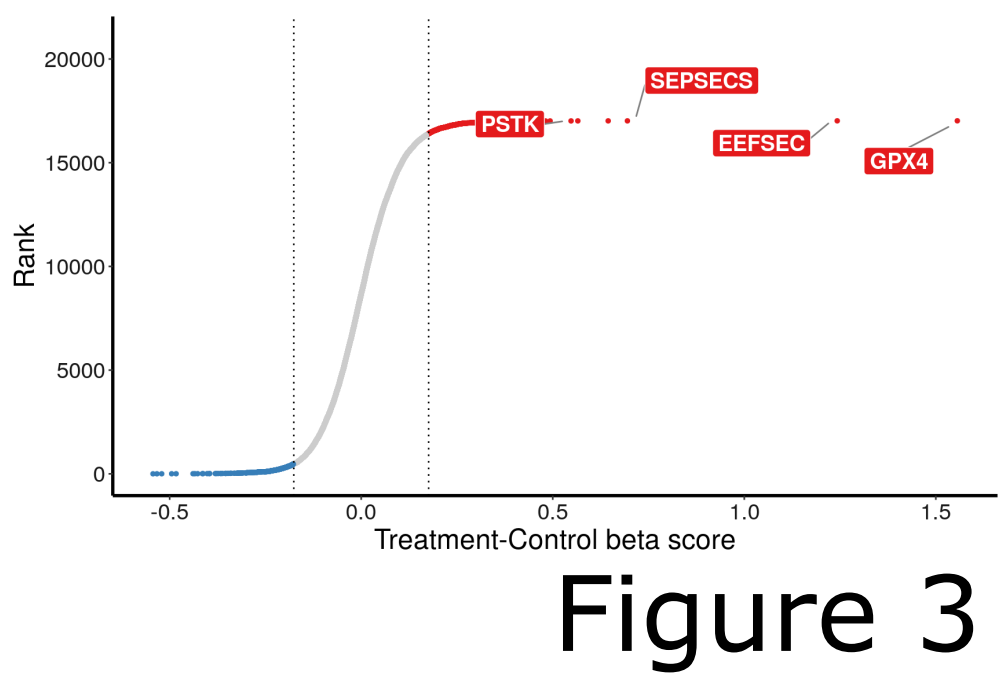


$\Delta \quad$ bioRxiv preprint doi: https://doi.org/10.1101/2022.02.01.478478; this version posted February 1,2022 . The copyright holder for this preprint (which was not certified by peer review) is the author/funder, who has granted bioRxiv a license to display the preprint in perpetuity. It is made

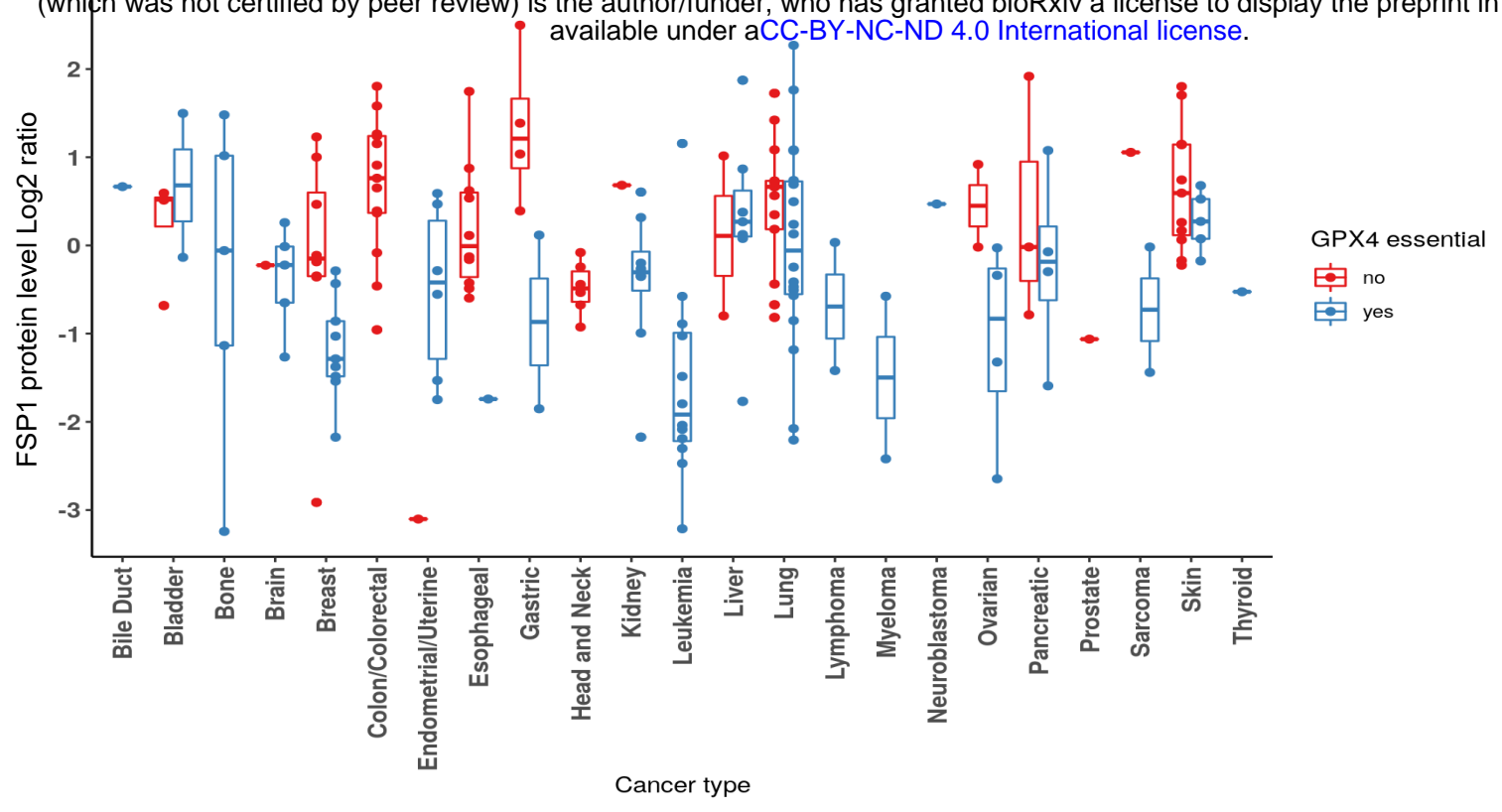

B

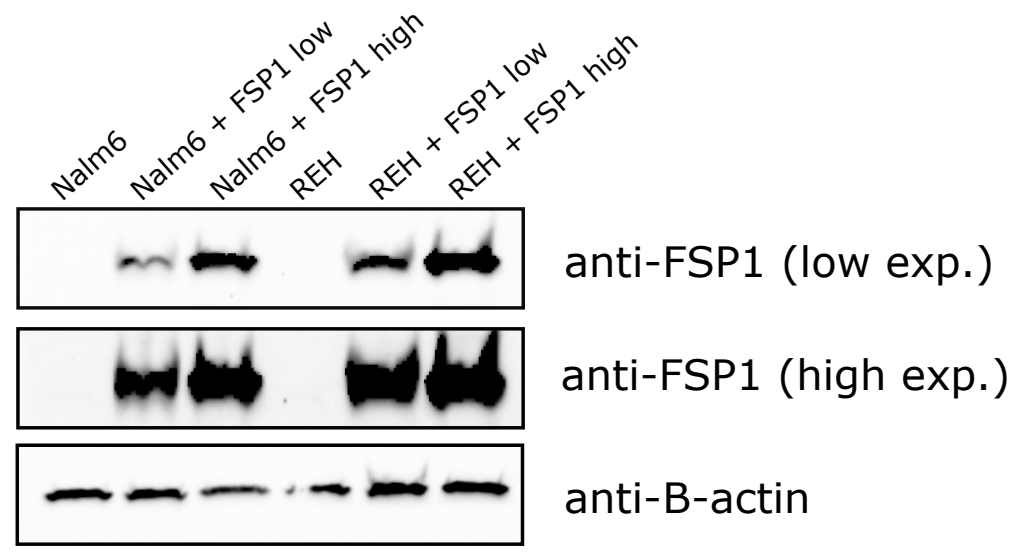

C

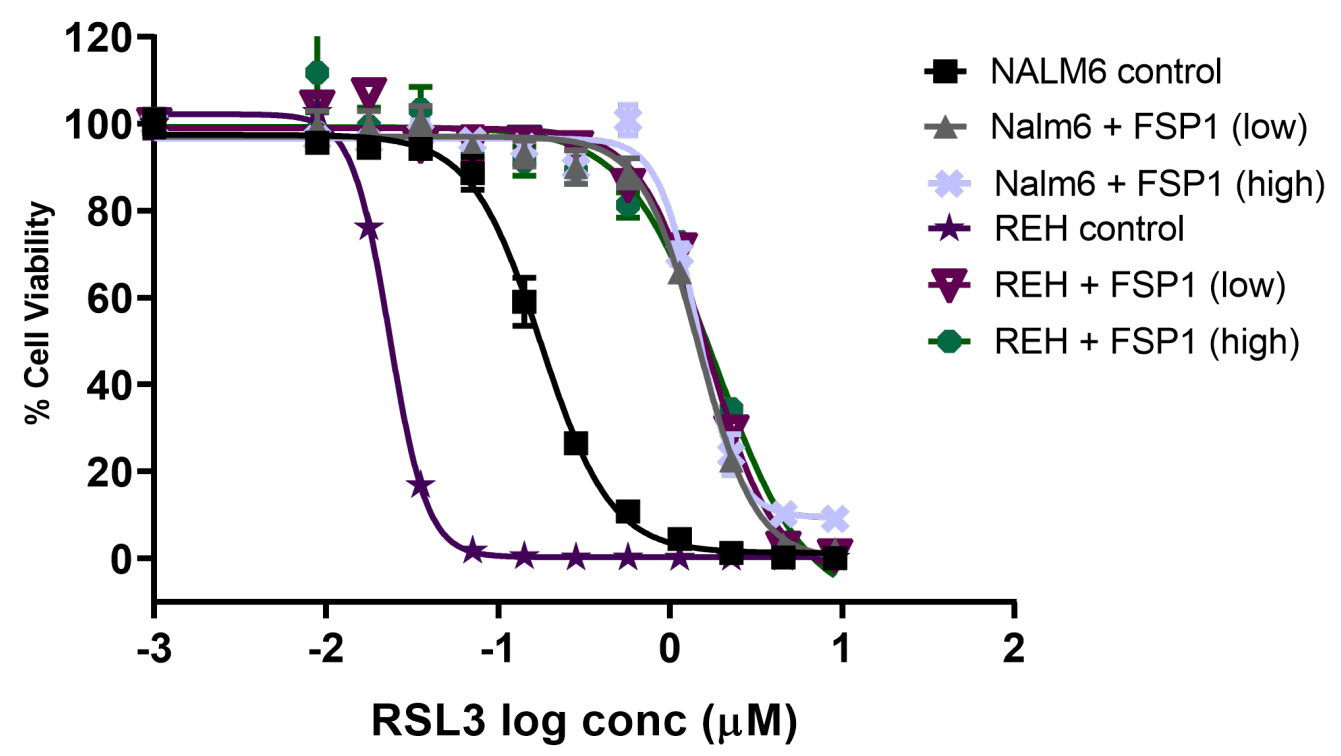

Figure 4 
A bioRxiv preprint doi: https://doi.org/10.1101/2022.02.01.478478; this vergimn posted February 1, 2022. The copyright holder for this preprint (which was not certified by peer review) is the author/funder, who has grarted bioRxiv a license to display the preprint in perpetuity. It is made

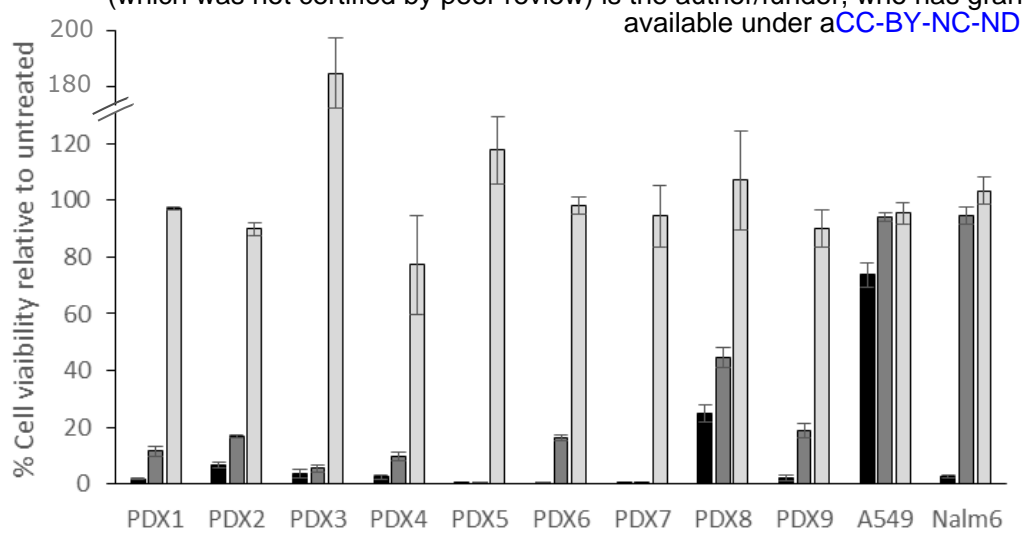
4.0 International license.

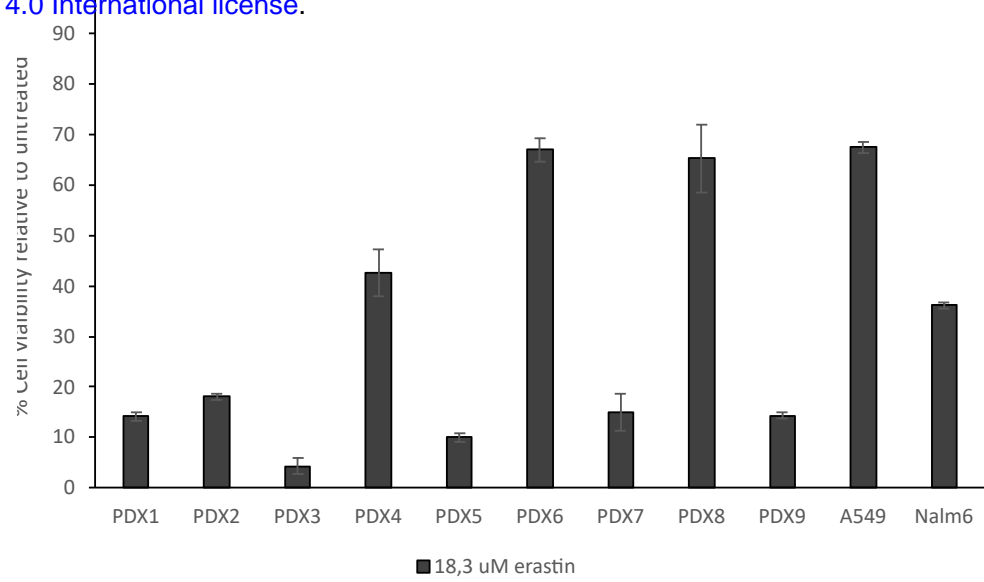

C

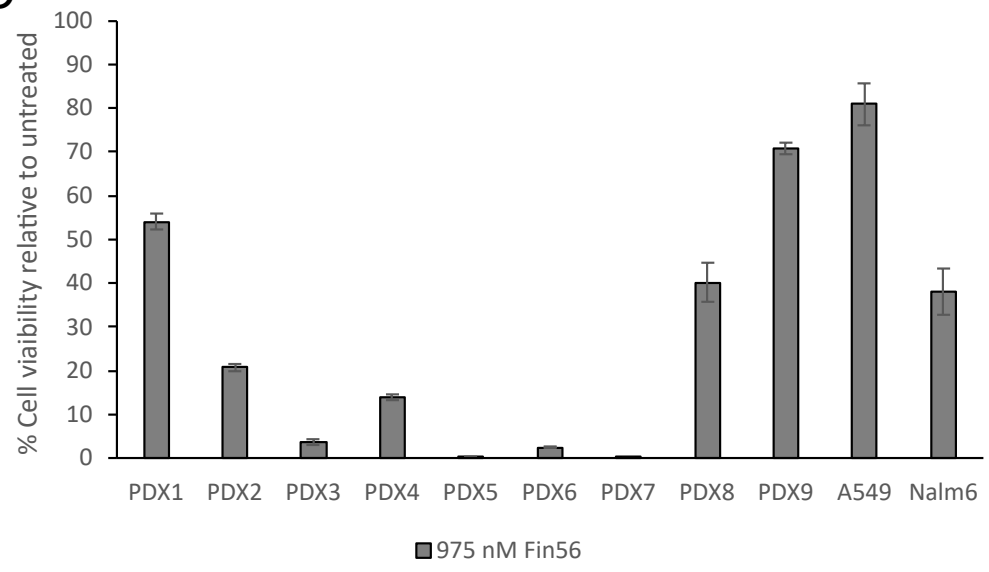

D

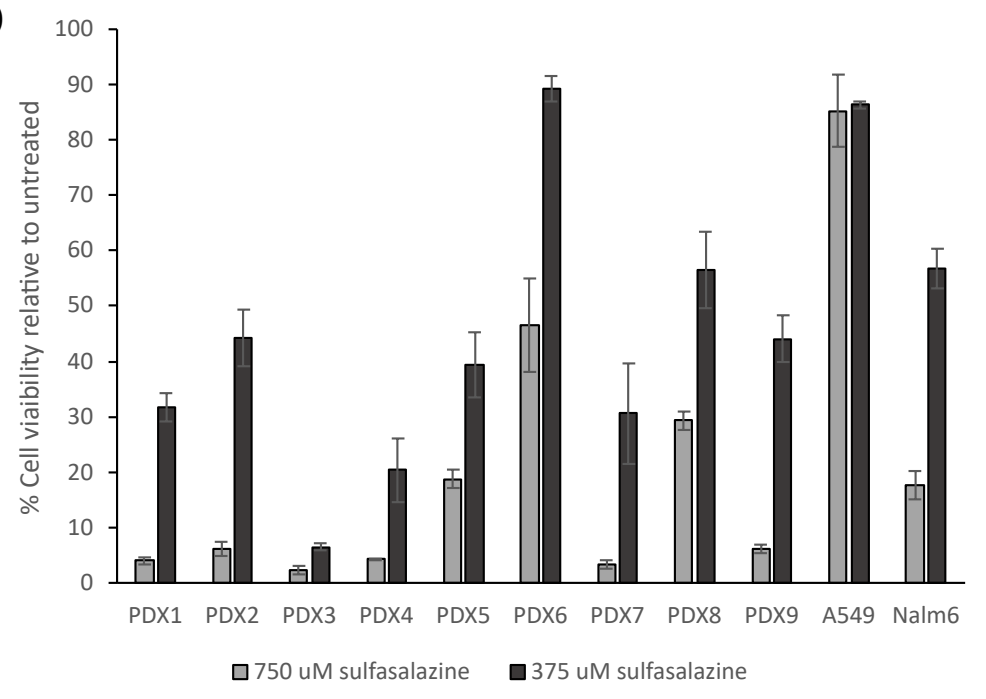


bioRxiv preprint doi: https://doi.org/10.1101/2022.02.01.478478. this version posted February 12022 . The copyright holder for this preprint (which was not certified by peer review) is the author/funder, who has granted bioRxiv a license to display the preprint in perpetuity. It is made available under aCC-BY-NC-ND 40 International license.

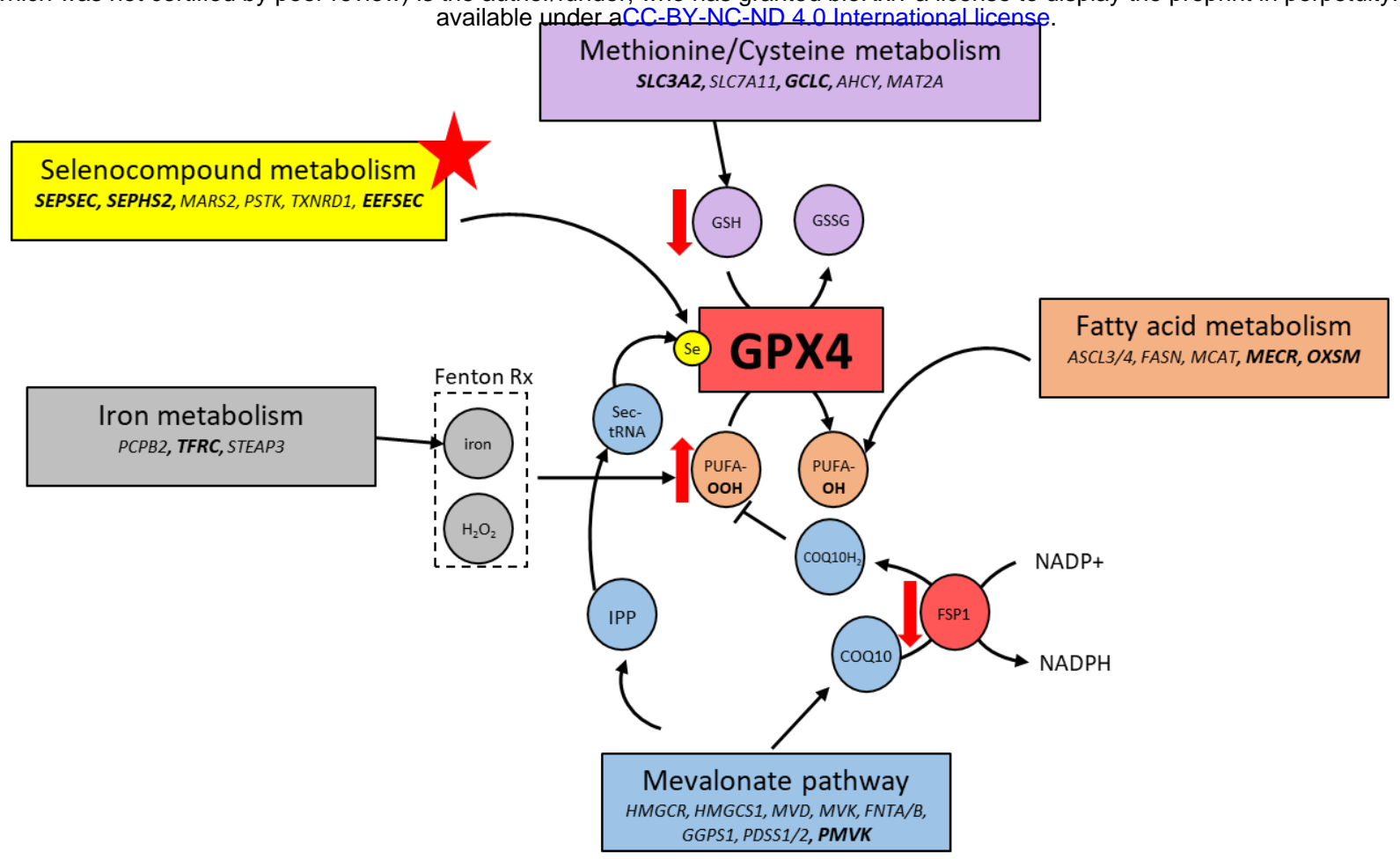

Figure 6 